\title{
The Antitumor Effect of Capsaicin on Prostate Cancer Cells Depends on AMPK Activation Through LKB1 Kinase and TRPV1 Receptor
}

\section{Belén G. Sánchez}

University of Alcalá

\section{Alicia Bort}

University of Alcalá

Ines Diaz-Laviada ( $\square$ ines.diazlaviada@uah.es)

University of Alcalá

\section{Research Article}

Keywords: TRPV1, LKB1, AMPK, capsaicin, PC3, LNCaP, DU-145, prostate cancer

Posted Date: August 26th, 2021

DOI: https://doi.org/10.21203/rs.3.rs-829520/v1

License: (c) (i) This work is licensed under a Creative Commons Attribution 4.0 International License.

Read Full License 


\section{Abstract}

Recently, natural compounds and their derivatives have been reported to have anti-cancer effects. Herein, we investigated the mechanism whereby the natural vanilloid capsaicin exerts anti-tumor effects on prostate cancer cells. We found that capsaicin activates the AMP-activated kinase (AMPK) and promotes cell death in the LKB1 expressing prostate cancer cell lines LNCaP and PC3 but not in the LKB1-null cell line DU-145. Capsaicin treatment stimulated LKB1 phosphorylation and activated AMPK in LKB1 expressing cells. In addition, overexpression of LKB1 by lentiviral infection in DU-145 cells, induced capsaicin-triggered AMPK activation and apoptosis while LKB1 silencing in LNCaP and PC3 cells, increased capsaicin-promoted cell death. Capsaicin-induced LKB1 phosphorylation, was dependent on the transient receptor potential cation channel subfamily V member 1 (TRPV1), since TRPV1 knocked down by shRNA, abolished LKB1 and AMPK phosphorylation and inhibited apoptosis. Altogether, our results show that capsaicin affect AMPK activity in an LKB1- and TRPV1-dependent fashion, linking TRPV1 with cell fate. These data also suggest that capsaicin may be a rational therapeutic option for prostate tumors.

\section{Introduction}

In the recent years, natural compounds used in combination with traditional chemotherapeutics to enhance their effectiveness have gained notable attention to develop new therapeutic interventions in cancer, as they can selectively target numerous signaling pathways implicated in tumor development and progression ${ }^{1}$. In this line, we have shown that the spicy ingredient extracted from peppers belonging to Solanaceae family, capsaicin, exert antitumor effects in prostate cancer, acting synergistically with docetaxel to inhibit prostate cancer growth ${ }^{2-4}$. Capsaicin has been shown to inhibit prostate cancer cells growth more efficacious than other natural compounds ${ }^{5}$. Capsaicin induces autophagy blockage and apoptosis in prostate cancer PC-3 cells ${ }^{4,6}$, inhibits the growth of castration-resistant prostate cancer cells 7,8 and causes the degradation of the androgen receptor (AR) ${ }^{9}$. In addition, it sensitized human prostate cancer cells to radiotherapy ${ }^{10}$ and reduces metastasis in the transgenic adenocarcinoma of the mouse prostate model ${ }^{11}$. Although capsaicin targets the transient receptor potential cation channel subfamily $\mathrm{V}$ member 1 (TRPV1) and inhibits the oxidoreductase tNOX ${ }^{12}$ the underpinning mechanism involved on its antiproliferative effect in prostate cells remains elusive ${ }^{13}$. We recently determined that the tumor suppressor properties and synergic efficacy of capsaicin were associated with the AMP-activated kinase (AMPK) activation ${ }^{2,14}$ but the mechanism used by capsaicin to activate AMPK remains unknown.

AMPK has been revealed as a relevant target in cancer with both beneficial and adverse roles ${ }^{15}$. Evidence supporting the beneficial role of AMPK activation in prostate cancer comes from patients with type 2 diabetes mellitus treated with metformin, an activator of AMPK $[52,53]$. Patients treated with metformin had a lower prostate cancer incidence ${ }^{16,17}$ and better response in survival and recurrence ${ }^{18}$. A negative association between serum PSA levels and metformin use has also been observed in prostate cancer patients ${ }^{19}$. In prostate cells, metformin suppresses androgen receptor activation and signaling pathways 
involved in cell growth and proliferation ${ }^{20}$. AICAR, another well-known activator of AMPK, induces apoptosis, inhibits migration and invasion of prostate cancer cells ${ }^{21}$ and sensitizes cells to radiotherapy 22. Likewise, nummularic acid extracted from traditional medicinal plants ${ }^{23}$ and $\mathrm{CO}^{24}$, inhibit prostate cancer cells growth through AMPK activation, pointing to a relevant therapeutic role of AMPK in prostate cancer. When AMPK is activated, it switch on catabolic pathways and switch off ATP-consuming processes leading to inhibition of cell growth and proliferation ${ }^{15}$. However, the role of AMPK in cancer is controversial since although it has been extensively demonstrated that AMPK activation protects from cancer incidence and behaves as a tumor suppressor, in some cases it can sustain cell growth and protect against the metabolic stress that undergo cancer cells ${ }^{15}$.

AMPK can be activated by canonical and non-canonical pathways ${ }^{25}$. The canonical pathway includes the increase in the AMP/ATP or ADP/ATP ratios and phosphorylation of the catalytic a subunit by the liver kinase B1 (LKB1) ${ }^{26}$, whereas the non-canonical mechanisms involve phosphorylation by other kinases, oxidative modification or binding of long-chain fatty acyl-CoA esters ${ }^{25}$. Phosphorylation at Thr172 of the AMPK a-subunit is regulated by at least four kinases namely, LKB1, calcium-/calmodulindependent kinase kinase 2 (CaMKK2), TGF $\beta$-activated kinase 1 (TAK1) and the recently proposed mixedlineage kinase 3 (MLK3) ${ }^{27}$. By other hand, AMP binds to the regulatory y subunit and allosterically enhances the phosphorylation of AMPK by LKB1 and inhibits dephosphorylation by protein phosphatases ${ }^{15}$.

In this study, we explored the involvement of LKB1 in the mechanism whereby capsaicin induces AMPK activation in prostate cancer cells. Our results show that capsaicin could significantly inhibit proliferation and induce apoptosis in human prostate cancer cell lines which express LKB1 but not in DU145 which do not express LKB1. Investigation of the underlying mechanism reveals an involvement of the receptor TRPV1 in the activation of the LKB1/AMPK axe.

\section{Results}

We first investigated the antiproliferative effect of capsaicin on the prostate cancer cell lines LNCaP, PC3 and DU-145. As shown in Figure 1A, capsaicin reduced the cell viability of the three cell lines, but was less potent in DU-145 cells, specially at the higher doses $80 \mathrm{mM}$ and $160 \mathrm{mM}$. The resistance of DU-145 cells to capsaicin was more clearly observed in apoptosis. While capsaicin induced $27 \%$ apoptosis in LNCaP cells and 18\% in PC3 cells, it failed to increase apoptosis in DU-145 cells (Figure 1B).

It has been described that the prostate DU-145 cell line harbors a loss-of-function mutation in the STK11 gene encoding LKB1 ${ }^{28}$. In consequence, we wonder whether the anti-survival effect induced by capsaicin was dependent on LKB1. Therefore, we examined the ability of capsaicin to stimulate LKB1 in prostate cells. Treatment of LNCaP and PC3 cells with capsaicin, sightly increased LKB1 phosphorylation in Ser428 which is indicative of its activation (Figure 2A). According to previously described, DU-145 cells did not express LKB1 and therefore, capsaicin did not induce its phosphorylation (Figure 2A). It is worthy 
to note that capsaicin produced a notable increase of AMPK phosphorylation in the LKB1 expressing cell lines LNCaP and PC3, while failed to activate AMPK in the LKB1-null cell line DU-145 (Figure 2A). These results indicate that capsaicin activates AMPK in a LKB1-depenent fashion. To corroborate this notion, we examined the phosphorylation of the AMPK downstream target Acetyl-CoA carboxylase (ACC), a key marker for determining AMPK activity in intact cells. As shown in figure 2A, ACC phosphorylation was increased in capsaicin-treated LNCaP and PC3 cells, but not in capsaicin-treated DU-145 cells. These findings support the notion that LKB1 plays a critical role in the mechanism whereby capsaicin activates AMPK in prostate cells.

To further investigate the involvement of LKB1 in capsaicin-induced AMPK activation, we knocked down LKB1 by siRNA in LNCaP and PC3 cells and assessed the ability of capsaicin to activate AMPK. As expected, phosphorylation of AMPK was blocked in capsaicin treated LKB1 downregulated cells (Figure $2 B$ ). Since we have demonstrated that capsaicin inhibits cell proliferation of prostate cells through AMPK ${ }^{2}$, and activation of AMPK by capsaicin relies on LKB1, then LKB1 silencing should inhibit capsaicin antiproliferative effect. We effectively confirmed that capsaicin-reduced cell viability was counteracted by LKB1 depletion (Figure 2C).

It has been stablished that AMPK regulates autophagy by indirectly inhibiting the mammalian target of rapamycin (mTOR) complex I. We have previously demonstrated that in prostate cells, capsaicin promotes autophagosome formation but inhibits the autophagic flux, causing an accumulation of the cargo protein $\mathrm{p} 62$ in the autophagolysosomes ${ }^{4}$. Since prostate cancer cells rely on autophagy activation for survival, autophagy blockage inhibits cell proliferation and induces cell death. Hence, we explored whether capsaicin regulation of autophagy was dependent on LKB1. As shown in Figure 2D, treatment of LNCaP and PC3 cells with capsaicin, promoted an inhibition of mTOR phosphorylation and induced an increase in the lipid-modified form of LC3-II indicating an activation of autophagy, as previously described. Capsaicin treatment of LNCaP and PC3 cells, also promoted an accumulation of p62, indicative of an autophagy flux blockage (Figure 2D). Interestingly, LKB1 knock down remarkably reduced p62 accumulation as well as mTOR inhibition in both cell lines and LC3-II increase in PC3 cells (Figure 2D). These results indicate that LKB1 is involved in the autophagy regulation produced by capsaicin in prostate cells.

As a further proof of the involvement of LKB1 in AMPK activation by capsaicin, we stably overexpressed LKB1 in DU-145 cells by lentiviral infection with particles carrying a Lenti_Neo_LKB1 vector. LKB1 overexpression was confirmed by western blotting analyses (Figure 3A). Interestingly, capsaicin was able to activate AMPK in LKB1 expressing DU-145 cells. Treatment of DU-145 infected cells with $80 \mathrm{mM}$ capsaicin induced AMPK and ACC phosphorylation, both phenomena indicative of AMPK activation (Figure 3A).

We next investigate whether capsaicin inhibited cell proliferation following re-expression of LKB1 in LKB1-null tumor cell lines. As shown in Figure 3B, the reintroduction of LKB1 into DU-145 cells, increased 
capsaicin-induced cell death and apoptosis and reduced the number of cells in G0/G1 phase in a fashion similar to PC3 cells (supplementary figure 1).

\section{CaMKK2 is not involved in AMPK activation induced by capsaicin in prostate cells}

In addition to LKB1, AMPK can also be activated by the upstream kinase CaMKK2 in prostate cells ${ }^{29}$. To investigate the involvement of this kinase in the capsaicin-induced activation of AMPK, we use the selective and cell-permeable pharmacological CaMKK2 inhibitor ST0-609. As shown in Figure 4, the pretreatment with ST0-609 failed to prevent the phosphorylation of AMPK or ACC induced by capsaicin in PC3 cells. Intriguingly, STO-609 totally abolished the basal and stimulated phosphorylation of either AMPK or ACC in the LKB1-null cell line DU-145 (Figure 4). These results indicate that the CaMKK2 is not involved in the action mechanism whereby capsaicin activates AMPK in prostate cells. However, CaMKK2 might be involved in the basal phosphorylation of AMPK in DU-145 cells which do not express LKB1.

\section{TRPV1 is required for LKB1 and AMPK activation}

Capsaicin effectively activates the transient receptor potential vanilloid 1 (TRPV1), a cation channel expressed in sensitive neurons as well as in other tissues including prostate and prostate cancer cells ${ }^{30,31}$. It has been recently discovered that TRPV1 plays essential roles in cancer tumorigenesis and development ${ }^{32}$. However, TRPV1 agonists may exert antitumor effects via a receptor dependent or independent mechanism ${ }^{32}$. To examine the involvement of the TRPV1 channel in the mechanism of capsaicin-induced anti-tumor effect and AMPK activation, we knocked down TRPV1 expression by infection with lentiviral viruses carrying small hairpin RNA (shRNA). The most efficient sequence against TRPV1, was cloned into the lentiviral vector pLKO.1 and the resulting plasmid was used to produce viruses in HEK293T cells. Empty vector viruses (pLKO.1 EV) were used to infect control cells. The knockdown efficiency of TRPV1-specific shRNA was confirmed at protein level by Western blotting analyses through comparison with those of a negative control (Figure 5A). Genetic downregulation of TRPV1 abolished the expression of LKB1 and the increase in AMPK phosphorylation induced by capsaicin in PC3 cells (Figure 5A). As expected, in DU-145 cells, genetic depletion of TRPV1 did not modify the phosphorylation of AMPK in the presence of capsaicin (Figure 5A). This shows that TRPV1 is required for LKB1 and AMPK activation highlighting an TRPV1/LKB1/AMPK signaling pathway in prostate cancer cells.

Genetic depletion of TRPV1 slightly but significantly inhibited the capsaicin-induced decrease of cell viability (Figure 5B). Moreover, TRPV1 silencing had an impact on the induction of apoptosis by capsaicin. In PC3-TRPV1-shRNA-infected cells, a significant prevention in capsaicin-induced apoptosis was observed (Figure 5B). Nevertheless, in DU-145 cells, TRPV1 downregulation did not have any impact on apoptosis, according to the lack of effectiveness of capsaicin on this cell line (Figure 5B). Finally, we 
analyzed cell cycle by flow cytometry. As shown in figure 5C, treatment of prostate PC3 cells with capsaicin resulted in diminution of cells at the G0/G1 phase and accumulation in the G2/M phase. Then again, TRPV1 knocked down, blocked the cell cycle arrest promoted by capsaicin in PC3 cells. These results indicate that the anti-proliferative effect induced by capsaicin in prostate cells is mediated by a TRPV1-LKB1-AMPK dependent mechanism.

\section{Discussion}

Here, we demonstrate that capsaicin exerts antiproliferative effects in prostate cancer cells expressing LKB1 by a TRPV1 receptor dependent mechanism. Capsaicin reduces cell viability, promotes apoptosis and induces cell cycle arrest in G2/M, by activation of the TRPV1/LKB1/AMPK axe. It has been recently revealed that TRPV1 is involved in cancer development and progression although the precise role that TRPV1 plays remains to be elucidated. Despite both the expression and activity of TRPV1 are altered in many tumors, there is still great confusion about its role in regulating cell fate. In fact, both agonists and antagonists may reveal anti-cancer effects, and the effect may function via or be independent of TRPV1 32. For instance, Amantini et al. have demonstrated that treatment of urothelial cancer cells with capsaicin arrests cell cycle progression and triggers apoptosis through a TRPV1-dependent fashion ${ }^{33}$. In human renal carcinoma, capsaicin induces apoptosis that is reversed by the TRPV1 antagonist capsazepine, implying a receptor-mediated mechanism ${ }^{34}$. However, in hepatocarcinoma cells, Zhang et al. demonstrated that capsaicin enhances antitumor activity of sorafenib independently of TRPV $1^{35}$. We have previously demonstrated that capsaicin exerts synergistic antitumor effect with sorafenib in hepatocellular carcinoma cells and with docetaxel in prostate cancer cells through AMPK activation 2,36. Our results in this study show that capsaicin activates AMPK in prostate cancer cells via TRPV1/LKB1dependent phosphorylation at Thr172.

Due to the important role of AMPK in regulating cell fate and metabolism, the phosphorylation of AMPK Thr172 is tightly regulated by upstream kinases and phosphatases. However, the predominant upstream AMPK kinase in the prostate has not been well stablished ${ }^{37}$. Although the role of the CaMKK2 in prostate cancer has been recently revealed, it does not appear to have an essential effect on growth regulation ${ }^{38}$. CaMKK2 levels have been found to be elevated in clinical samples of prostate cancer where it regulates cancer cell growth ${ }^{39}$. In addition, androgens regulate the expression of CaMKK2 in prostate cells, harbouring the CaMKK2 promoter, an androgen responsive element ${ }^{38,40}$. However, protein synthesis is unperturbed by targeting the AR-CaMKK2-AMPK pathway in prostate cancer cells, suggesting that CaMKK2, although stimulating glycolysis has not significant effects on biosynthesis ${ }^{38}$. This agrees with our results showing that AMPK activation by capsaicin is independent of CaMKK2. On the contrary, our results show that capsaicin activates AMPK in prostate cells by a LKB1-dependent pathway that regulates cell growth.

Shackelford et al. demonstrated several years ago that the drug phenformin, a biguanide chemically related with metformin, was more effective in the treatment of non-small cell lung cancer (NSCLC) if the 
tumors lack a functional LKB1-AMPK pathway, suggesting a LKB1-AMPK-independent mechanism of action ${ }^{41}$. However, this is not the case in our work, since capsaicin inhibited cell growth more efficiently in PC3 and LNCaP cells than in DU145 cells, which lack LKB1. Moreover, the rescue of LKB1 expression by lentiviral infection in DU-145 cells, allowed AMPK activation and cell growth inhibition by capsaicin. The activation of AMPK by LKB1 in prostate LNCaP and PC3 cells has been also observed by Yan et al. ${ }^{24}$ who described that the treatment of prostate cells with CO, provoked an increase in LKB1 expression and AMPK activation and significantly suppressed tumor growth.

Our work describes a novel connection between the capsaicin receptor TRPV1 and the LKB1/AMPK pathway in prostate cells. Our novel finding that TRPV1 acts as upstream regulator of LKB1 uncovers a molecular pathway linking the cation channel with cell fate. In agreement with our results, Li et al. recently proposed that TRPV channels may activate AMPK independently of AMP and that genetic depletion of TRPV1 blocks AMPK activation which is indicative of physical requirement of TRPV1 to activate AMPK ${ }^{42}$. TRPV1 channel induces lysosomal AMPK activation in low glucose through the formation of an AXIN-based super-complex on the lysosomal surface, that allows LKB1 to phosphorylate and activate AMPK. According to Maiese K. ${ }^{43}$, TRPV1 receptors do not rely entirely upon calcium signaling to affect cellular biology, but also have a close relationship with AMPK, mTOR and protein kinase B (Akt), which agrees with our results. Here, we show that TRPV1 is connected with AMPK via LKB1, although we do not know the mechanism whereby TRPV1 is linked to LKB1 in prostate cells. Further research to unravel the underlying pathway will shed light on the role of TRPV1 in growth regulation.

Altogether our results indicate that the activation of TRPV1/LKB1/AMPK pathway by capsaicin resulted in a significant decrease in cell proliferation, suggesting that TRPV1 targeted pharmaceutical interventions could be exploited to suppress the growth of prostate tumors.

\section{Methods}

\section{Materials.}

Capsaicin (CAP) and STO-609 were purchased to Sigma-Aldrich (St. Louis, MO, USA). psPAX2 vector was a gift from Didier Trono (Addgene plasmid \#12260; http://n2t.net/addgene:12260;

RRID:Addgene_12260), pCMV-VSV-G was a gift from Robert Weinberg ${ }^{44}$ (Addgene plasmid \#8454; http://n2t.net/addgene:8454; RRID:Addgene_8454), pLKO.1-TRC cloning vector was a gift from from David Root 45 (Addgene plasmid \#10878; http://n2t.net/addgene:10878; RRID:Addgene_10878), pMDLg/pRRE was a gift from Didier Trono ${ }^{46}$ (Addgene plasmid \#12251; http://n2t.net/addgene:12251; RRID:Addgene_12251), pRSV-Rev was a gift from Didier Trono ${ }^{46}$ (Addgene plasmid \#12253; http://n2t.net/addgene:12253; RRID:Addgene_12253) and LentiV_Neo_LKB1 was a gift from Christopher Vakoc ${ }^{47}$ (Addgene plasmid \#108111; http://n2t.net/addgene:108111; RRID: Addgene_108111). 


\section{Cell culture.}

PC3, DU-145 and LNCaP human prostate cancer cell lines were obtained from American Type Culture Collection, (ATCC CRL-1435, ATCC HTB-81 and ATCC CRL-1740 respectively) (Rockville, MD, USA). Cells were routinely grown in RPMI 1640 medium supplemented with $100 \mathrm{lU} / \mathrm{ml}$ penicillin $\mathrm{G}$ sodium, $100 \mathrm{mg} / \mathrm{ml}$ streptomycin sulfate, $0.25 \mathrm{mg} / \mathrm{ml}$ amphotericin B (Invitrogen, Paisley, UK) and $10 \%$ fetal bovine serum. All cell lines were incubated at $37^{\circ} \mathrm{C}$ in $5 \% \mathrm{CO}_{2}$ and routinely tested for Mycoplasma infection. For treatment experiments, cells were plated and grown $24 \mathrm{~h}$, the medium was then replaced with serum-free RPMI 1640 and then incubated with different treatments for the indicated times. Cells were used at passages 4-20.

\section{Cell viability.}

Cell proliferation/viability was determined by the MTT assay (Bio-Rad, Richmond, CA, USA). The assay was performed in 12-well plates, according to the manufacturer's instructions $\left(5 \times 10^{3} /\right.$ well). The absorbance was measured at 490 and $650 \mathrm{~nm}$ using an iMark ${ }^{\mathrm{TM}}$ Absorbance Reader from Bio-Rad (Richmond, CA, USA).

\section{Flow cytometry for cell cycle and apoptosis.}

Flow cytometry was used to detect the distribution of cell cycle. After being cultivated with the treatment, $1.5 \cdot 10^{5}$ cells in $35 \mathrm{~mm}$ culture dish were harvested in $0.35 \%$ trypsin, collected and washed with cold PBS. After that, cells were centrifuged at $500 \mathrm{~g}$ for $5 \mathrm{~min}$ and fixed with $70 \%$ cold ethanol at $-20^{\circ} \mathrm{C}$ overnight. Then, cells were centrifuged again and incubated in $0.5 \mathrm{ml}$ PBS containing $0.1 \mathrm{mg} / \mathrm{ml}$ RNase for $30 \mathrm{~min}$ at $37^{\circ} \mathrm{C}$. DNA staining was performed by adding $10 \mu \mathrm{g} / \mathrm{ml} \mathrm{PI} \mathrm{(Invitrogen,} \mathrm{Eugene,} \mathrm{Oregon,} \mathrm{USA).}$ Apoptosis was evaluated at $24 \mathrm{~h}$ following treatment using an Annexin V-fluorescein isothiocyanate (FITC) Apoptosis Detection kit BD Biosciences, San Diego, CA USA) according to the manufacturer's instructions. Data acquisition and analysis were performed in a MACSQuant ${ }^{\circledR}$ Analyzer flow cytometry system (Miltenyi Biotec, Bergisch Gladbach, Germany) using MACSQuantify software (Miltenyi Biotec, Bergisch Gladbach, Germany). A total of $10 \times 10^{3}$ events were collected for each sample.

\section{Western blot.}

Proteins for Western blotting were isolated by lysing cells in lysis buffer [50 mM Tris pH 7.4, $0.8 \mathrm{M} \mathrm{NaCl}, 5$ $\mathrm{mM} \mathrm{MgCl} 2,0.1 \%$ Triton X-100] containing protease inhibitor and phosphatase inhibitor cocktail (Roche, Diagnostics; Mannheim, Germany), incubated on ice for $15 \mathrm{~min}$ and cleared by microcentrifugation. 20 micrograms of total protein/lane were separated by SDS-polyacrylamide gel electrophoresis (SDS-PAGE) and then transferred onto a PVDF membrane. Membranes were incubated overnight at $4^{\circ} \mathrm{C}$ with primary antibodies. After washing in T-TBS, membranes were incubated with peroxidase-conjugated anti-mouse 
or anti-rabbit secondary antibodies (1:5000) for $2 \mathrm{~h}$ at room temperature. The immune complex was visualized with an ECL system (Cell Signaling Technology). Protein expression levels were quantified using Image $J$ (National Institutes of Health, Bethesda, Maryland, USA) expressed as fold changes relative to the control treatment. Primary antibodies anti-p62, p-AMPKa1-thr172, p-ACC-ser79, pLKB1ser428, pmTOR-ser2448 and the antibodies against the corresponding total forms were obtained from Cell Signaling Technology (Danvers, MA, USA). TRPV1 was obtained from (Thermo Scientific, MA, USA) and LC3B was from Novus (England, UK). Peroxidase labeled secondary anti-mouse IgG was from SigmaAldrich (St. Louis, MO, USA) and anti-rabbit IgG was from Cell Signaling Technology (Danvers, MA, USA).

\section{siRNA transfections.}

Cells were transfected in $1 \mathrm{~mL}$ OptiMEM containing $4 \mu \mathrm{g}$ Lipofectamine iMax (Invitrogen, Carlsbad, CA) with 100 nM LKB1-specific small interfering RNA (siRNA) duplexes (5'-GUACUUCUGUCAGCUGAUUdTdT-3' and 5'-AAUCAGCUGACAGAAGUACdTdT-3') (Sigma-Aldrich, St. Louis, MO, USA) or scrambled RNA (control) according to the manufacturer's protocols (Invitrogen). At $72 \mathrm{~h}$ after transfection, the medium was removed and replaced with RPMI. At the indicated time points after transfection, cells were used for MTT cell viability assays or western blot analysis.

\section{Lentivirus transduction}

The lentiviral transduction system was used to generate cell lines with TRPV1 silencing or LKB1 overexpression. Lentivirus was produced in HEK293T cells by transfecting plasmids of interest with helper plasmids. To generate the viruses to silence TRPV1, the following mixture was added to a $10 \mathrm{~cm}$ dish of HEK293T at 70\% confluence: $5 \mu \mathrm{g}$ of psPAX2, $3 \mu \mathrm{g}$ of pCMV-VSV-G, $10 \mu \mathrm{g}$ of pLKO.1-TRC cloning vector or pLKO.1-TRC cloning vector with shTRPV1 (shTRPV1 sequence was designed from the clone ID: TRCN0000044190, Sigma, St. Louis, MO, USA) and polythylenimine (PEI) 1mg/ml at a 3:1 ratio with the total concentration of the DNA in the mix. On the other hand, to generate the viruses to overexpress LKB1, the mixture was the following: $5 \mu \mathrm{g}$ of pMDLg/pRRE, $3 \mu \mathrm{g}$ of pCMV-VSV-G, $2.5 \mu \mathrm{g}$ of pRSV-Rev, $10 \mu \mathrm{g}$ of plasmid LentiV_Neo_LKB1 and PEl $1 \mathrm{mg} / \mathrm{ml}$ in the same relationship discussed above. At 6 hours after transfection, the medium was changed to fresh medium and after $48 \mathrm{~h}$ and $72 \mathrm{~h}$ after transfection, the supernatant with the viruses was collected, filtered through a $0.45 \mu \mathrm{m}$ pore-size filter and used to infect PC3 and DU-145 cells, adding polybrene $(1 \mu \mathrm{g} / \mathrm{ml})$ (Sigma, St. Louis, MO, USA) to increase the efficiency

of the infection. After infection, the cells were amplified to a larger culture surface and $24 \mathrm{~h}$ later they were selected with $3 \mu \mathrm{g} / \mathrm{mL}$ puromycin (STEMCELL Technologies) in the case of TRPV1 silencing or with $900 \mu \mathrm{g} / \mathrm{ml}$ G418 (Sigma, St. Louis, MO, USA) in the case of LKB1 overexpression.

\section{Statistical analysis}


The statistical analysis of the results was performed with Graph Pad Prism 9 software (San Diego, CA) using a two-way ANOVA and Tukey's multiple comparisons test or Sidak's multiple comparisons test. The results were reported as mean \pm SD as indicated in figure caption, of at least three independent experiments. Data were considered significant when $p \leq 0.05$.

\section{Abbreviations}

AMPK, AMP-activated kinase; CaMKK2, calcium-/calmodulin-dependent kinase kinase 2; LKB1, liver kinase B1; TRPV1, Transient receptor potential vanilloid type 1

\section{Declarations}

\section{Acknowledgments}

Authors acknowledge the technical assistance of Natalia Huertas and NANOBIOSIS.

\section{Funding}

This research was financially supported by the by Instituto de Salud Carlos III through the project "PI20/01327" (Co-funded by European Regional Development Fund "A way to make Europe") and by Fundación Tatiana Pérez de Guzmán el Bueno (Grant Patrocinio 2019-001).

\section{Data Availability}

The data used to support the findings of this study are deposited in https://data.mendeley.com/datasets/w77g7vdsjp/draft?a=92c84270-6569-4d39-bed4-9278ca2257fe.

\section{Conflicts of Interest}

All authors declare no conflicts of interest.

\section{Authors' Contributions}

BGS carried out most of the experiments, performed the analytic calculations and contributed to the interpretation of the results. $A B$ participated in the project design as well as manuscript draft preparation and revision. ID-L conceived the project and wrote the manuscript. All authors read and approved the final manuscript.

\section{References}


1. Fontana, F., Raimondi, M., Marzagalli, M., Di Domizio, A. \& Limonta, P. Natural Compounds in Prostate Cancer Prevention and Treatment: Mechanisms of Action and Molecular Targets., 9, https://doi.org/10.3390/cells9020460 (2020).

2. Sanchez, B. G., Bort, A., Mateos-Gomez, P. A., Rodriguez-Henche, N. \& Diaz-Laviada, I. Combination of the natural product capsaicin and docetaxel synergistically kills human prostate cancer cells through the metabolic regulator AMP-activated kinase. Cancer Cell Int, 19, 54 https://doi.org/10.1186/s12935-019-0769-2 (2019).

3. Diaz-Laviada, I. \& Rodriguez-Henche, N. The potential antitumor effects of capsaicin. Prog Drug Res, 68, 181-208 (2014).

4. Ramos-Torres, A., Bort, A., Morell, C., Rodriguez-Henche, N. \& Diaz-Laviada, I. The pepper's natural ingredient capsaicin induces autophagy blockage in prostate cancer cells. Oncotarget, 7, 1569-1583 https://doi.org/10.18632/oncotarget.6415 (2016).

5. Fort, R. S. et al. Isolation and Structural Characterization of Bioactive Molecules on Prostate Cancer from Mayan Traditional Medicinal Plants. Pharmaceuticals (Basel), 11, https://doi.org/10.3390/ph11030078 (2018).

6. Sanchez, A. M., Sanchez, M. G., Malagarie-Cazenave, S., Olea, N. \& Diaz-Laviada, I. Induction of apoptosis in prostate tumor PC-3 cells and inhibition of xenograft prostate tumor growth by the vanilloid capsaicin., 11, 89-99 https://doi.org/10.1007/s10495-005-3275-z (2006).

7. Mori, A. et al. Capsaicin, a component of red peppers, inhibits the growth of androgen-independent, p53 mutant prostate cancer cells. Cancer Res, 66, 3222-3229 https:// doi.org/10.1158/00085472.CAN-05-0087 (2006).

8. Sanchez, A. M. et al. Apoptosis induced by capsaicin in prostate PC-3 cells involves ceramide accumulation, neutral sphingomyelinase, and JNK activation., 12, 2013-2024 https://doi.org/10.1007/s10495-007-0119-z (2007).

9. Zheng, L. et al. Capsaicin causes inactivation and degradation of the androgen receptor by inducing the restoration of miR-449a in prostate cancer. Oncol Rep, 34, 1027-1034 https://doi.org/10.3892/or.2015.4055 (2015).

10. Venier, N. A. et al. Capsaicin: a novel radio-sensitizing agent for prostate cancer., 75, 113-125 https://doi.org/10.1002/pros.22896 (2015).

11. Venier, N. A. et al. Capsaicin reduces the metastatic burden in the transgenic adenocarcinoma of the mouse prostate model., 75, 1300-1311 https://doi.org/10.1002/pros.23013 (2015).

12. Axanova, L., Morre, D. J. \& Morre, D. M. Growth of LNCaP cells in monoculture and coculture with osteoblasts and response to tNOX inhibitors. Cancer Lett, 225, 35-40 https://doi.org/10.1016/j.canlet.2004.11.017 (2005).

13. Ziglioli, F. et al. Vanilloid-mediated apoptosis in prostate cancer cells through a TRPV-1 dependent and a TRPV-1-independent mechanism. Acta Biomed, 80, 13-20 (2009).

14. Bort, A. et al. Targeting AMP-activated kinase impacts hepatocellular cancer stem cells induced by long-term treatment with sorafenib. Mol Oncol, 13, 1311-1331 https://doi.org/10.1002/1878- 
0261.12488 (2019).

15. Vara-Ciruelos, D., Russell, F. M. \& Hardie, D. G. The strange case of AMPK and cancer: Dr Jekyll or Mr Hyde? (dagger). Open Biol, 9, 190099 https://doi.org/10.1098/rsob.190099 (2019).

16. Kuo, Y. J. et al. Metformin reduces prostate cancer risk among men with benign prostatic hyperplasia: A nationwide population-based cohort study. Cancer Med, 8, 2514-2523 https://doi.org/10.1002/cam4.2025 (2019).

17. Wang, Y. et al. Effect of metformin on the risk of prostate cancer in patients with type 2 diabetes by considering different confounding factors: a meta-analysis of observational studies. Eur J Cancer Prev, https://doi.org/10.1097/CEJ.0000000000000514 (2019).

18. Taussky, D. et al. Impact of diabetes and metformin use on prostate cancer outcome of patients treated with radiation therapy: results from a large institutional database. Can J Urol, 25, 9509-9515 (2018).

19. Park, J. S., Lee, K. S., Ham, W. S., Chung, B. H. \& Koo, K. C. Impact of metformin on serum prostatespecific antigen levels: Data from the national health and nutrition examination survey 2007 to 2008. Med. (Baltim), 96, e9427 https://doi.org/10.1097/MD.0000000000009427 (2017).

20. Zaidi, S., Gandhi, J., Joshi, G., Smith, N. L. \& Khan, S. A. The anticancer potential of metformin on prostate cancer. Prostate Cancer Prostatic Dis, https://doi.org/10.1038/s41391-018-0085-2 (2019).

21. Su, C. C. et al. AICAR Induces Apoptosis and Inhibits Migration and Invasion in Prostate Cancer Cells Through an AMPK/mTOR-Dependent Pathway. Int J Mol Sci, 20, https://doi.org/10.3390/ijms20071647 (2019).

22. Rae, C. \& Mairs, R. J. AMPK activation by AICAR sensitizes prostate cancer cells to radiotherapy. Oncotarget, 10, 749-759 https://doi.org/10.18632/oncotarget.26598 (2019).

23. Younis, T. et al. Nummularic acid, a triterpenoid, from the medicinal plant Fraxinus xanthoxyloides, induces energy crisis to suppress growth of prostate cancer cells. Mol Carcinog, 57, 1267-1277 https://doi.org/10.1002/mc.22841 (2018).

24. Yan, Y. et al. CO suppresses prostate cancer cell growth by directly targeting LKB1/AMPK/mTOR pathway in vitro and in vivo. Urol Onco/36, 312 e311-312 e318, doi:10.1016/j.urolonc.2018.02.013 (2018).

25. Russell, F. M. \& Hardie, D. G. AMP-Activated Protein Kinase: Do We Need Activators or Inhibitors to Treat or Prevent Cancer? Int J Mol Sci, 22, https://doi.org/10.3390/ijms22010186 (2020).

26. Hardie, D. G. \& Lin, S. C. AMP-activated protein kinase - not just an energy sensor. F1000Res, 6, 1724 https://doi.org/10.12688/f1000research.11960.1 (2017).

27. Luo, L., Jiang, S., Huang, D., Lu, N. \& Luo, Z. MLK3 phophorylates AMPK independently of LKB1. PLoS One, 10, e0123927 https://doi.org/10.1371/journal.pone.0123927 (2015).

28. Ikediobi, O. N. et al. Mutation analysis of 24 known cancer genes in the $\mathrm{NCl}-60$ cell line set. $\mathrm{Mol}$ Cancer Ther, 5, 2606-2612 https://doi.org/10.1158/1535-7163.MCT-06-0433 (2006). 
29. Popovics, P., Frigo, D. E., Schally, A. V. \& Rick, F. G. Targeting the 5'-AMP-activated protein kinase and related metabolic pathways for the treatment of prostate cancer. Expert Opin Ther Targets, 19, 617632 https://doi.org/10.1517/14728222.2015.1005603 (2015).

30. Wang, H. P., Pu, X. Y. \& Wang, X. H. Distribution profiles of transient receptor potential melastatinrelated and vanilloid-related channels in prostatic tissue in rat. Asian J Androl, 9, 634-640 https://doi.org/10.1111/j.1745-7262.2007.00291.x (2007).

31. Prevarskaya, N., Zhang, L. \& Barritt, G. TRP channels in cancer. Biochim Biophys Acta, 1772, 937946 https://doi.org/10.1016/j.bbadis.2007.05.006 (2007).

32. Li, L. et al. The Impact of TRPV1 on Cancer Pathogenesis and Therapy: A Systematic Review. Int J Biol Sci, 17, 2034-2049 https://doi.org/10.7150/ijbs.59918 (2021).

33. Amantini, C. et al. Triggering of transient receptor potential vanilloid type 1 (TRPV1) by capsaicin induces Fas/CD95-mediated apoptosis of urothelial cancer cells in an ATM-dependent manner., 30, 1320-1329 https://doi.org/10.1093/carcin/bgp138 (2009).

34. Liu, T. et al. Capsaicin mediates caspases activation and induces apoptosis through P38 and JNK MAPK pathways in human renal carcinoma. BMC Cancer, 16, 790 https://doi.org/10.1186/s12885016-2831-y (2016).

35. Zhang, S. S. et al. Capsaicin enhances the antitumor activity of sorafenib in hepatocellular carcinoma cells and mouse xenograft tumors through increased ERK signaling. Acta Pharmacol Sin, 39, 438-448 https://doi.org/10.1038/aps.2017.156 (2018).

36. Bort, A., Spinola, E., Rodriguez-Henche, N. \& Diaz-Laviada, I. Capsaicin exerts synergistic antitumor effect with sorafenib in hepatocellular carcinoma cells through AMPK activation. Oncotarget, 8, 87684-87698 https://doi.org/10.18632/oncotarget.21196 (2017).

37. Khan, A. S. \& Frigo, D. E. A spatiotemporal hypothesis for the regulation, role, and targeting of AMPK in prostate cancer. Nat Rev Urol, 14, 164-180 https://doi.org/10.1038/nrurol.2016.272 (2017).

38. Massie, C. E. et al. The androgen receptor fuels prostate cancer by regulating central metabolism and biosynthesis. EMBO J, 30, 2719-2733 https://doi.org/10.1038/emboj.2011.158 (2011).

39. Fu, $\mathrm{H}$. et al. MicroRNA-224 and its target CAMKK2 synergistically influence tumor progression and patient prognosis in prostate cancer. Tumour Biol, 36, 1983-1991 https://doi.org/10.1007/s13277014-2805-0 (2015).

40. Frigo, D. E. et al. CaM kinase kinase beta-mediated activation of the growth regulatory kinase AMPK is required for androgen-dependent migration of prostate cancer cells. Cancer Res, 71, 528-537 https://doi.org/10.1158/0008-5472.CAN-10-2581 (2011).

41. Shackelford, D. B. Unravelling the connection between metabolism and tumorigenesis through studies of the liver kinase B1 tumour suppressor. J Carcinog, 12, 16 https://doi.org/10.4103/14773163.116323 (2013).

42. Li, M. et al. Transient Receptor Potential V Channels Are Essential for Glucose Sensing by Aldolase and AMPK. Cell Metab, https://doi.org/10.1016/j.cmet.2019.05.018 (2019). 
43. Maiese, K. Warming Up to New Possibilities with the Capsaicin Receptor TRPV1: mTOR, AMPK, and Erythropoietin. Curr Neurovasc Res, 14, 184-189 https://doi.org/10.2174/1567202614666170313105337 (2017).

44. Stewart, S. A. et al. Lentivirus-delivered stable gene silencing by RNAi in primary cells. RNA, 9, 493501 https://doi.org/10.1261/rna.2192803 (2003).

45. Moffat, J. et al. A lentiviral RNAi library for human and mouse genes applied to an arrayed viral highcontent screen., 124, 1283-1298 https://doi.org/10.1016/j.cell.2006.01.040 (2006).

46. Dull, T. et al. A third-generation lentivirus vector with a conditional packaging system. J Virol, 72, 8463-8471 https://doi.org/10.1128/JVI.72.11.8463-8471.1998 (1998).

47. Tarumoto, Y. et al. LKB1, Salt-Inducible Kinases, and MEF2C Are Linked Dependencies in Acute Myeloid Leukemia. Mol Cell 69, 1017-1027 e1016, doi:10.1016/j.molcel.2018.02.011 (2018).

\section{Figures}


A

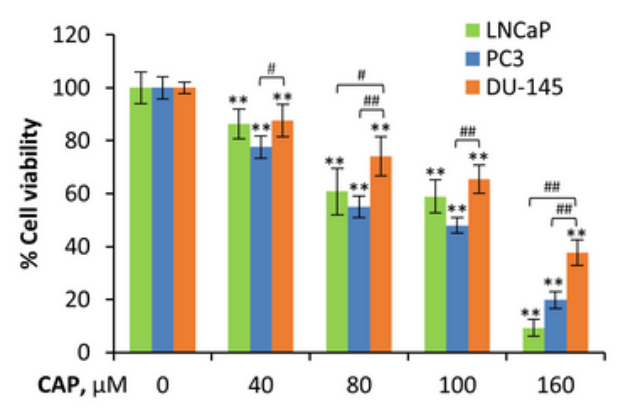

B
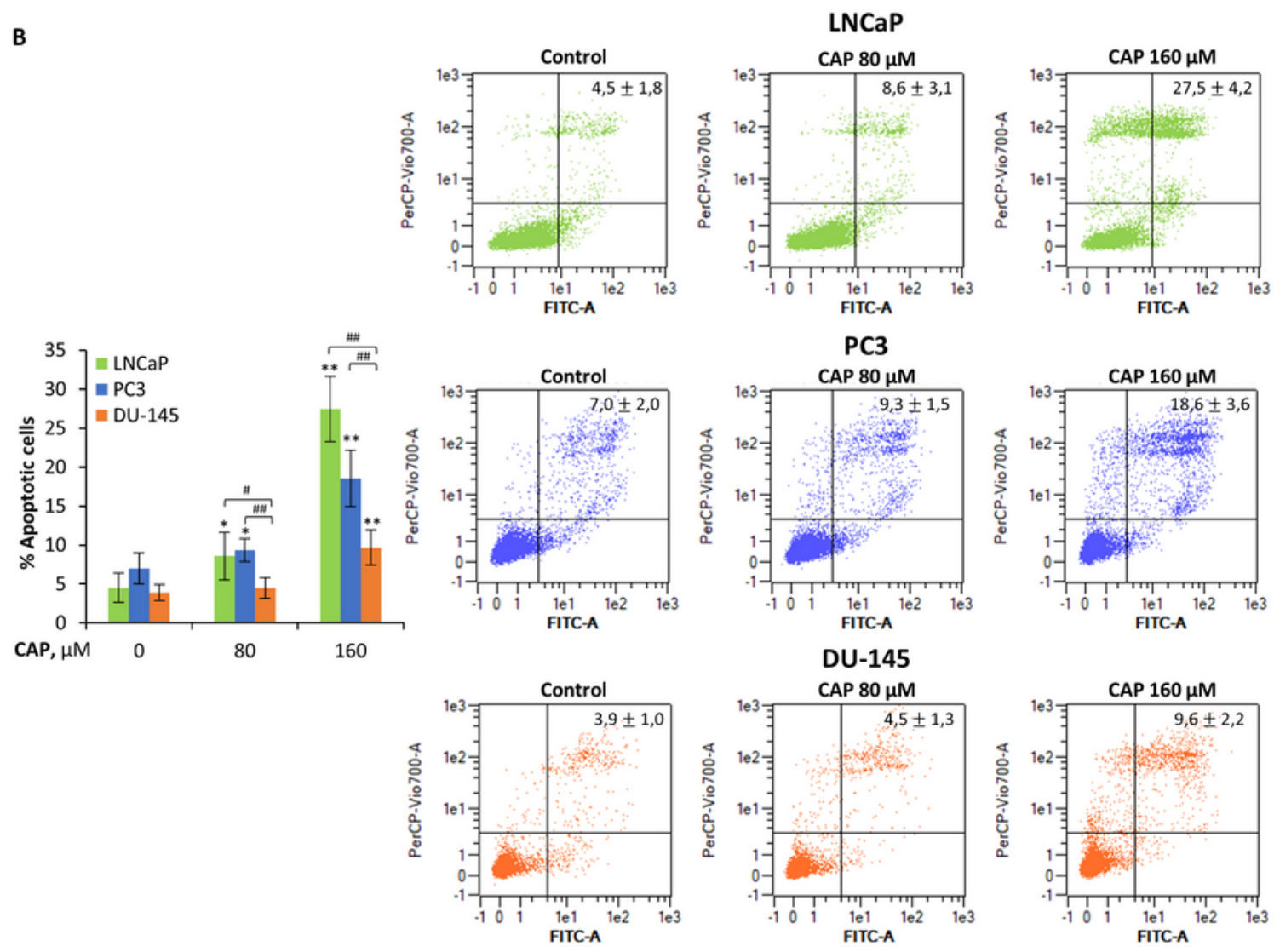

\section{Figure 1}

Lower antiproliferative effect of capsaicin on the prostate cancer cell line DU-145. A, Effect of the different doses of capsaicin on PCa cells viability. Cells were treated with capsaicin at the indicated concentrations for $24 \mathrm{~h}$. Cell viabilities were determined by MTT assay and expressed as percentages of control (DMSO treatment). B, LNCaP, PC3 and DU-145 cells were treated with DMSO (control) or the indicated doses of capsaicin for $24 \mathrm{~h}$ and then stained with Annexin $\mathrm{V}$ and $\mathrm{PI}$. The graphs represent PI 
fluorescence ( $\mathrm{Y}$ axe) versus Annexin V fluorescence ( $\mathrm{X}$ axe). The apoptotic cells (Annexin V-positive, PIpositive cells) are indicated as the percentage of gated cells. Bar graph represents the late apoptotic cells for each dose. Data are the mean $\pm S D .{ }^{*}, p<0.01$ and ${ }^{*}, p<0,0001$ significant difference between treated and control cells by two-way ANOVA and Tukey's multiple comparisons test; \#, $p<0.001$ and \#\#, $p<0,0001$ indicate significant difference between DU-145 and LNCaP or PC3. Experiments were run in triplicate and carried out at least five times on separate occasions.
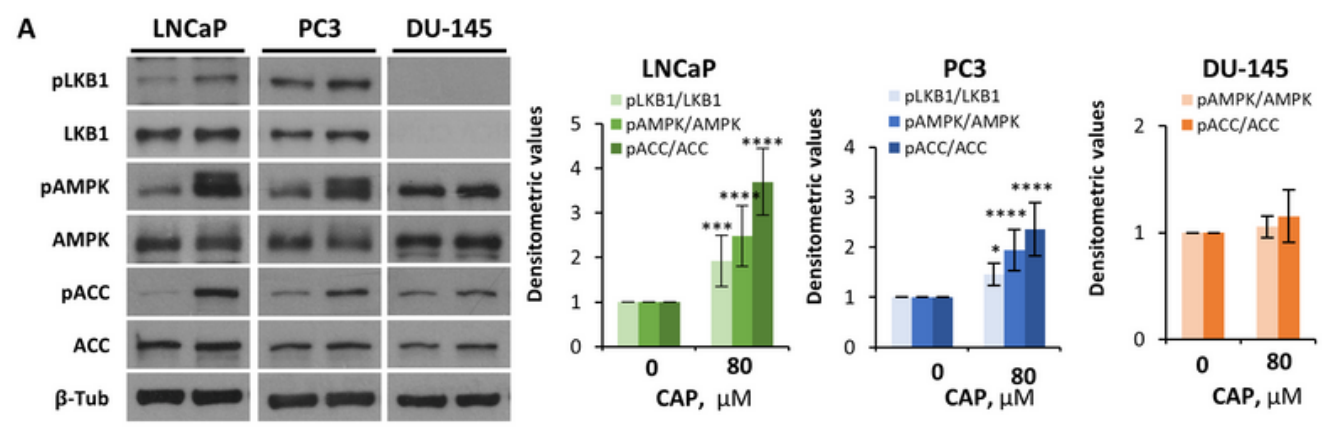

CAP, $80 \mu \mathrm{M}$ -
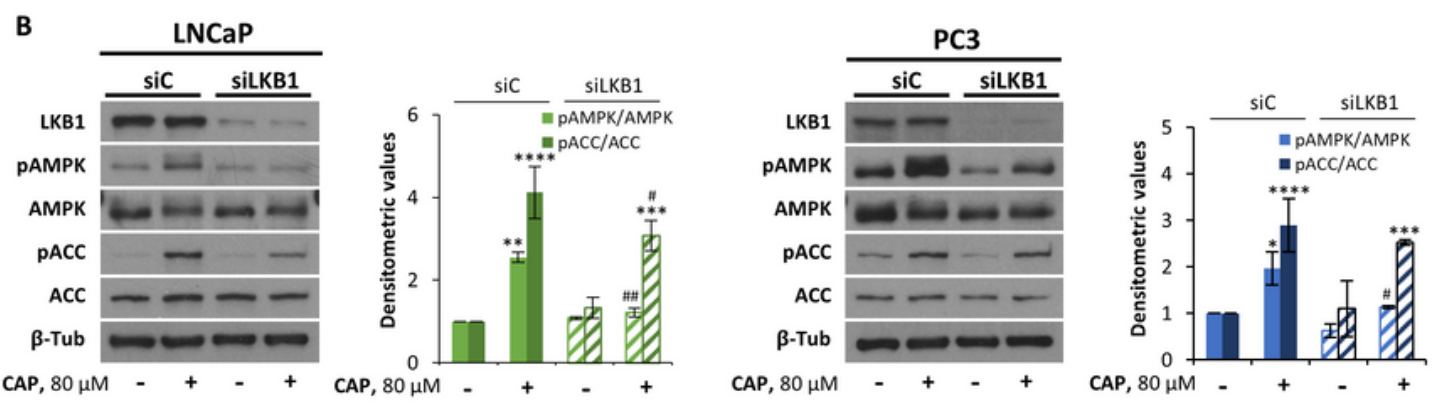

c
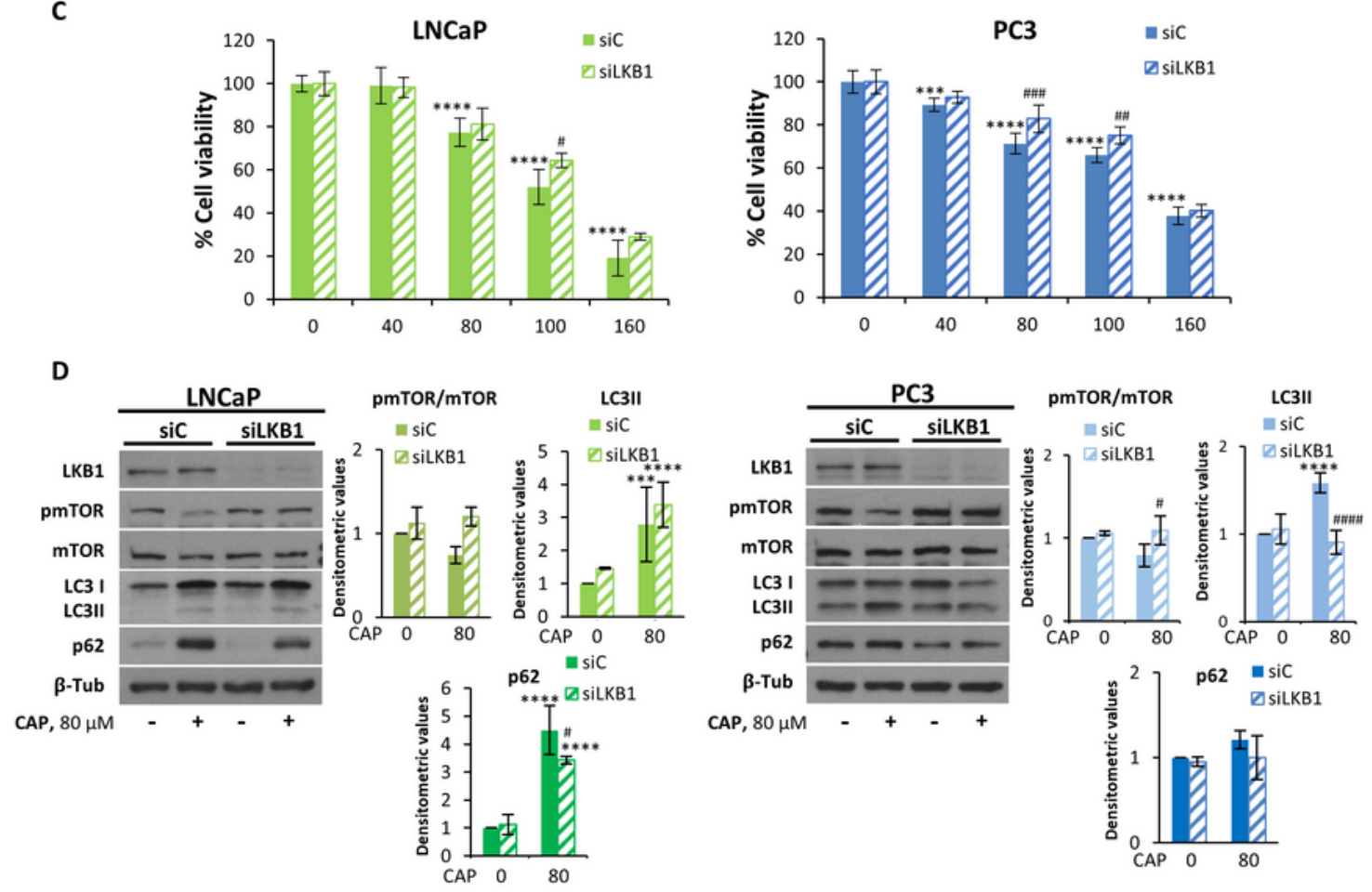

Figure 2 
Capsaicin-induced activation of the AMPK pathway is dependent on LKB1 expression. A, LNCaP, PC3 y DU-145 cells were treated with capsaicin for 1 hour. Levels of the phosphorylated proteins and their total forms were determined by Western blot and $\beta$-Tub served as a loading control. B, LNCaP and PC 3 cells were transfected with siControl $(\mathrm{siC})$ or selective siLKB1 for $72 \mathrm{~h}$ Then, cells were treated with $80 \mu \mathrm{M} \mathrm{CAP}$ during $1 \mathrm{~h}$. Levels of the phosphorylated proteins and their total forms were determined by Western blot and $\beta$-Tub served as a loading control. C, Effect of the LKB1 silencing by siRNA on cell viability. Cell viabilities were determined by MTT assay and expressed as percentages of those of control (DMSO treatment). Experiments were run in triplicate and carried out at least two times on separate occasions. D, $\mathrm{LNCaP}$ and PC3 cells were treated with $80 \mu \mathrm{M} \mathrm{CAP}$ during $24 \mathrm{~h}$. Levels of the proteins were determined by Western blot and $\beta$-Tub served as a loading control. A representative image of at least three experiments is shown. The densitometric analyses of bands are represented as the mean $\pm S D$. ${ }^{*} p<0.05,{ }^{\star \star} p<0,01$, $\star \star \star * p<0,001, * \star \star \star p<0,0001$ significant difference between treated cells regarding to the control (DMSO treatment) by two-way ANOVA and Tukey's multiple comparisons test; \#, $p<0.05, \# \#, p<0,01, \# \# \#, p<0,001$ significant difference between non-silenced and silenced cells by two-way ANOVA and Tukey's or Sidak's multiple comparisons test.
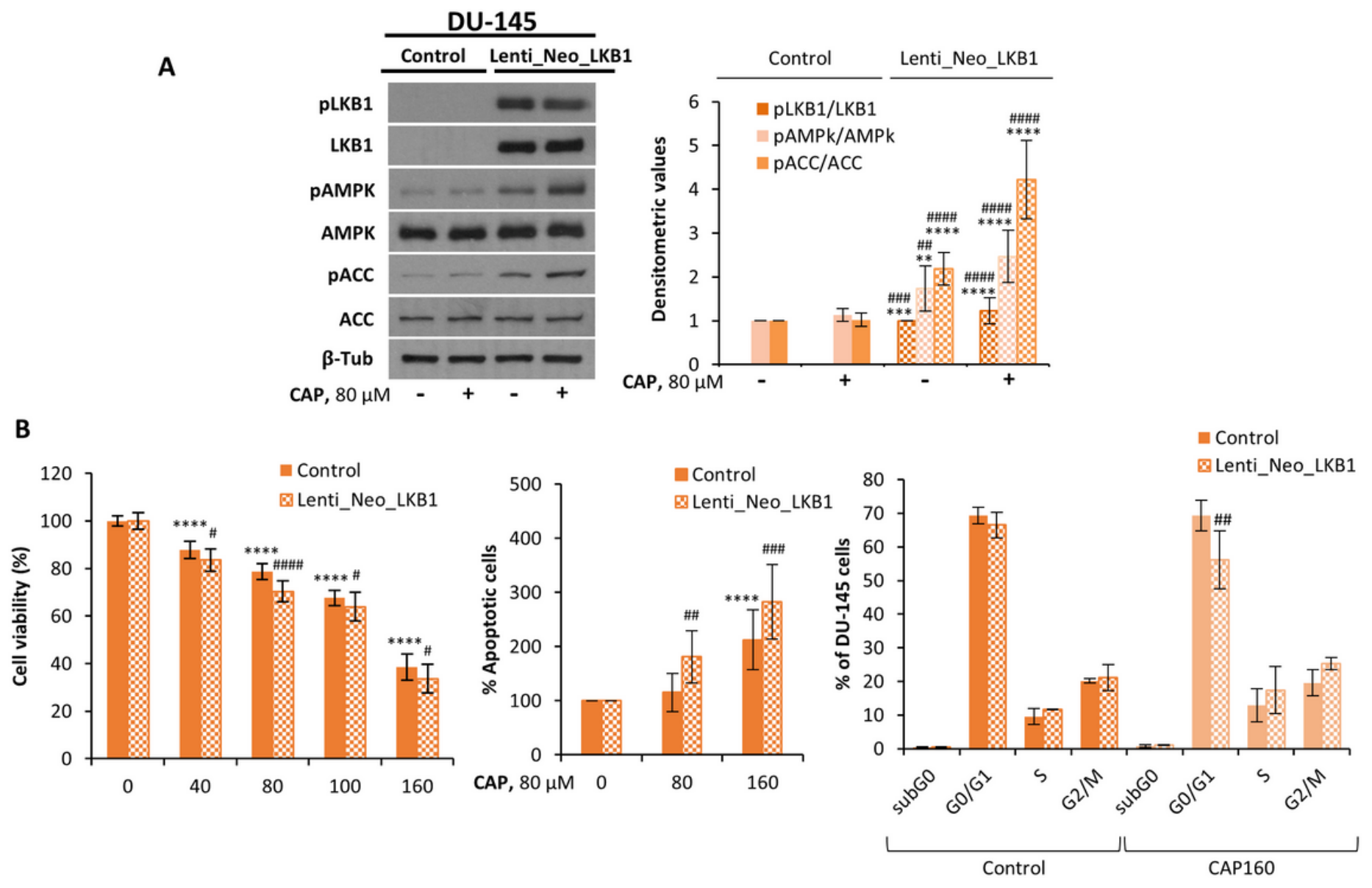

\section{Figure 3}


LKB1 expression in DU-145 cells promotes capsaicin-mediated activation of the LKB1/AMPK pathway. DU-145 cells were transfected with LKB1 by lentivirus-mediated transfection. A, Cells were treated with CAP during $1 \mathrm{~h}$. Levels of the proteins were determined by Western blot and $\beta$-Tub served as a loading control. The densitometric analyses of bands are represented as the mean \pm SD of five different experiments. B, Effect of the LKB1 overexpression on cell viability and apoptosis. Cell viabilities were determined by MTT assay and expressed as percentages of those of control (DMSO treatment). For apoptosis determination, DU-145 cells were treated with DMSO (control) or the indicated doses of capsaicin for $24 \mathrm{~h}$ and then stained with Annexin V and PI. Data are the mean \pm SD of eight different experiments. Data of the control of non-silenced and silenced cells appear normalized to 100\%. C, DU-145 cells were treated $24 \mathrm{~h}$ and cell cycle analysis was analyzed by flow cytometry using PI. Percentage of cells in subG0, G1, S, and G2/M phases is indicated in the bar graph. ${ }^{*} p<0.05,{ }^{*} p<0,01,{ }^{* \star *} p<0,001$, $* \star \star \star p<0,0001$ significant difference between treated cells regarding to the control (DMSO treatment) by two-way ANOVA and Tukey's multiple comparisons test; \#, $p<0.05$, \#\#, $p<0,01, \# \# \#, p<0,001$ significant difference between non-silenced and silenced cells by two-way ANOVA and Tukey's or Sidak's multiple comparisons test. 

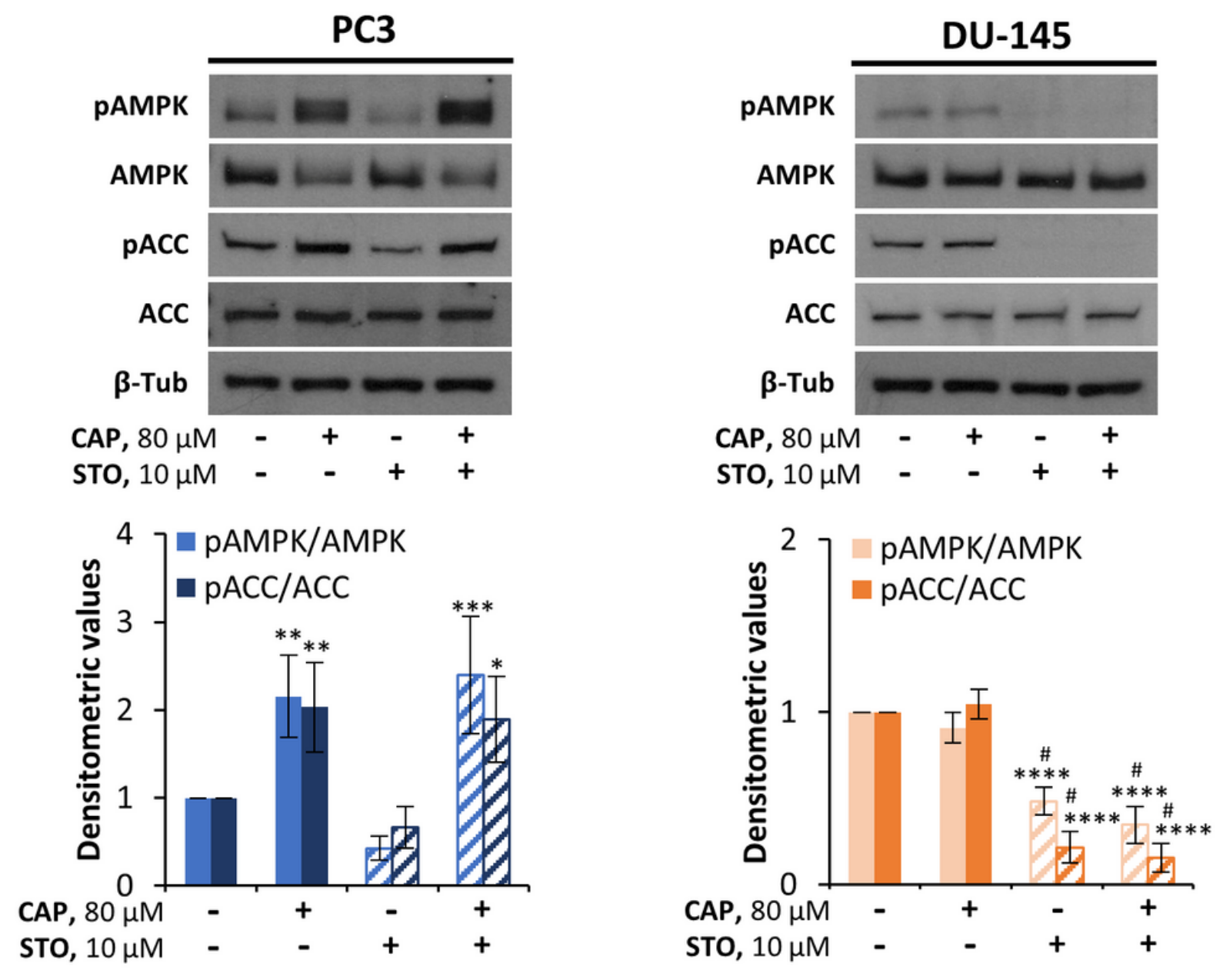

Figure 4

AMPK activation by capsaicin is independent of CaMKK2. PC3 and DU-145 cells were pretreated with 10 $\mu \mathrm{M}$ ST0-609 for half an hour and then incubated with $80 \mu \mathrm{M}$ CAP during $1 \mathrm{~h}$. Levels of proteins were determined by Western blot and $\beta$-Tubulin served as a loading control. The densitometric analyses of bands are represented as the mean \pm SD of four different experiments. ${ }^{*} p<0.05,{ }^{* \star} p<0,01,{ }^{\star \star *} p<0,001$, $\star \star \star \star p<0,0001$ significant difference between treated cells regarding to the control (DMSO treatment) by two-way ANOVA and Tukey's multiple comparisons test; \#, $\mathrm{p}<0.0001$ significant difference between cells pretreated with STO-609 and unpretreated by two-way ANOVA and Tukey's multiple comparisons test. 
A
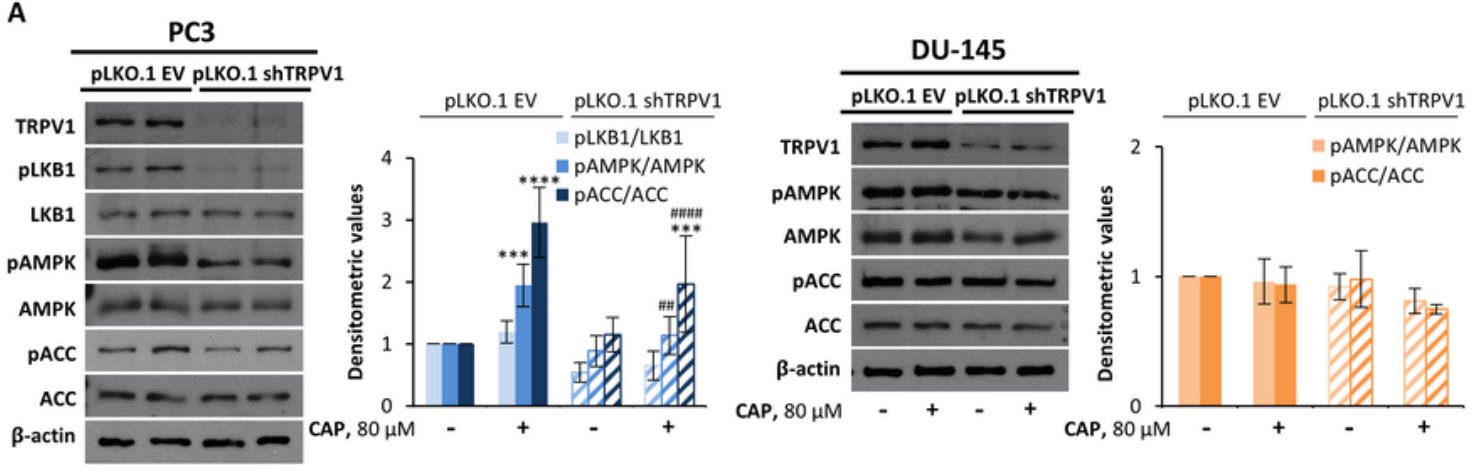

CAP, $80 \mu \mathrm{M}-+$

B
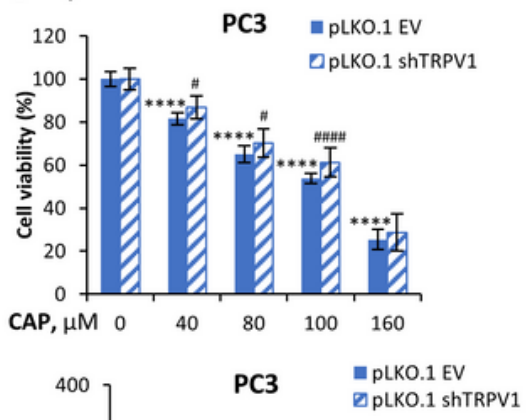

C

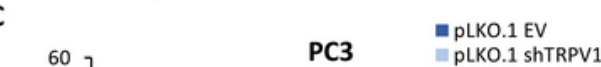

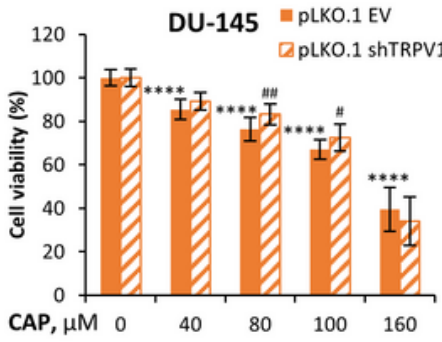

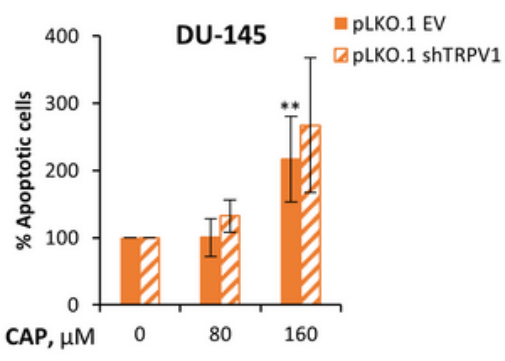

DU-145 = pLKO.1 EV

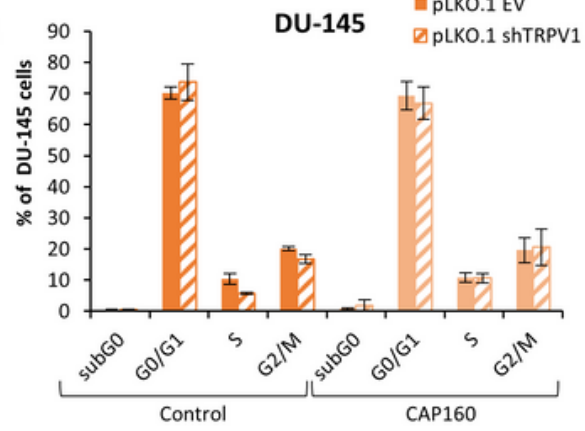

\section{Figure 5}

TRPV1 is required for LKB1 and AMPK activation. PC3 and DU-145 cells were transfected with shControl and shTRPV1 by lentivirus-mediated transfection. A. PC3 and DU-145 cells were treated with CAP during $1 \mathrm{~h}$. Levels of the proteins were determined by Western blot and $\beta$-actin served as a loading control. The densitometric analyses of bands are represented as the mean $\pm S D$ of five different experiments. $B$. Effect of the TRPV1 silencing on cell viability and apoptosis. Cell viabilities were determined by MTT assay and 
expressed as percentages of those of control (DMSO treatment). For apoptosis determination, PC3 and DU-145 cells were treated with DMSO (control) or the indicated doses of capsaicin for $24 \mathrm{~h}$ and then stained with Annexin V and PI. Data are the mean \pm SD of at least three different experiments. Data of the control of non-silenced and silenced cells appear normalized to $100 \%$ to appreciate the variations. C. PC3 and DU-145 cells were treated $24 \mathrm{~h}$ and cell cycle analysis was analyzed by flow cytometry using PI. Percentage of cells in subG0, G1, S, and G2/M phases is indicated in the bar graph. ${ }^{*} p<0.05$, ${ }^{*} p<0,01$, $\star * \star * p<0,001, * \star \star \star p<0,0001$ significant difference between treated cells regarding to the control (DMSO treatment) by two-way ANOVA and Tukey's multiple comparisons test; \#, $p<0.05, \# \#, p<0,01, \# \# \#, p<0,001$ significant difference between non-silenced and silenced cells by two-way ANOVA and Tukey's or Sidak's multiple comparisons test.

\section{Supplementary Files}

This is a list of supplementary files associated with this preprint. Click to download.

- Supplementaryfig1.tif 